\title{
Well-Posedness and Asymptotics for Initial Boundary Value Problems of Linear Relaxation Systems in One Space Variable
}

\author{
Shu-Yi Zhang and Ya-Guang Wang
}

\begin{abstract}
In this paper we study the well-posedness and relaxation limit for the initial boundary value problem of a general linear hyperbolic system with a relaxation term in one space variable. We mainly consider the asymptotic convergence and the boundary layer behavior under the sub-characteristic condition and the stiff Kreiss condition when the relaxation rate goes to zero, which generalizes the results of Xin and $\mathrm{Xu}$ in [J. Diff. Eqs. 167 (2000), 388 - 437] for homogeneous problems to the non-homogeneous case.
\end{abstract}

Keywords: Relaxation systems, initial-boundary value problems, boundary layers AMS subject classification: Primary 35L45, 35L50, secondary 35B40

\section{Introduction}

In this paper, we study the following initial-boundary value problem (IBVP) in the quarter plane $\{x>0, t>0\}$ for the linear form of Jin-Xin [3] relaxation system:

$$
\left\{\begin{aligned}
\partial_{t} u^{\epsilon}+\partial_{x} v^{\epsilon} & =q_{1}(x, t), \\
\partial_{t} v^{\epsilon}+a \partial_{x} u^{\epsilon} & =q_{2}(x, t)-\frac{1}{\epsilon}\left(v^{\epsilon}-f\left(u^{\epsilon}\right)\right), \\
u^{\epsilon}(x, 0) & =u_{0}(x) \\
v^{\epsilon}(x, 0) & =v_{0}(x), \\
B_{u} u^{\epsilon}(0, t)+B_{v} v^{\epsilon}(0, t) & =b(t),
\end{aligned}\right.
$$

where $\epsilon>0$ is the relaxation parameter, $a>0$ satisfies a sub-characteristic condition which will be given precisely later, $u^{\epsilon}, v^{\epsilon} \in R^{n}$ are vector-valued

Shu-Yi Zhang, P.O. Box A0207021, Shanghai Jiao Tong University, 200240 Shanghai, P.R.China; zhangshuyi@sjtu.edu.cn

Ya-Guang Wang, Department of Mathematics, Shanghai Jiao Tong University, 200030 Shanghai, P.R.China; ygwang@sjtu.edu.cn

ISSN 0232-2064 / \$2.50 C Heldermann Verlag Berlin 
unknowns, $B_{u}$ and $B_{v}$ are $n \times n$ constant real matrices, and $f(u)$ is linear, i.e.

$$
f(u)=F u
$$

for a $n \times n$ real constant matrix $F$. Furthermore, we assume that $F$ has n real eigenvalues and a complete set of eigenvectors, i.e. there are $n \times n$ matrices $L$ and $R$ such that

$$
L F R=\Lambda:=\operatorname{diag}\left\{\lambda_{1}, \ldots, \lambda_{n}\right\}, \quad L R=I_{n}
$$

Our main purpose is to study the boundary layer behaviors of the solution $\left(u^{\epsilon}, v^{\epsilon}\right)$ to the problem (1), and its asymptotic convergence to the solution of the corresponding equilibrium system

$$
\left\{\begin{aligned}
\partial_{t} u+\partial_{x} f(u) & =q_{1}(x, t), \\
v & =f(u)
\end{aligned}\right.
$$

when $\epsilon$ goes to zero.

Till now, there exists a rich literature devoted to the qualitative behaviors of solutions to the Cauchy problems of relaxation systems, e.g. refer to $[1,3-$ $5,8]$ and references therein. However, there are only a few rigorous theories on the asymptotic behaviors of solutions to the initial boundary value problems of relaxation systems when the relaxation parameter goes to zero, due to the complicated behaviors of the boundary layers in the process of the limits. This problem was studied by Wang and Xin in [6] for the scalar case of the limit equation. For the system case, in [9] Yong proposed the generalized Kreiss condition (GKC) for the well-posedness of both the relaxation system and the corresponding limit system, and rigorously studied the existence of the boundary layers without the initial layers. In the constant coefficient case, Xin and $\mathrm{Xu}$ [7] have established the well-posedness, the asymptotic behavior of boundary layers and initial layers for the homogeneous case of the problem (1). More precisely, in [7], it is required that for problem (1) that

$$
q_{1}(x, t)=q_{2}(x, t) \equiv 0
$$

and

$$
u_{0}(0)=v_{0}(0)=u_{0}^{\prime}(0)=v_{0}^{\prime}(0)=0, \quad b(0)=b^{\prime}(0)=0 .
$$

Here, we want to study the well-posedness of the problem (1) and the asymptotic behavior of the solutions $\left(u^{\epsilon}, v^{\epsilon}\right)$ when $\epsilon \rightarrow 0$ without the above restriction of $\mathrm{Xin}$ and $\mathrm{Xu}[7]$. As usual, we impose the following compatibility conditions on the problem (1):

$$
v_{0}(0)=F u_{0}(0), v_{0}^{\prime}(0)=F u_{0}^{\prime}(0), F q_{1}(0,0)-q_{2}(0,0)=F v_{0}^{\prime}(0)-a u_{0}^{\prime}(0)
$$


and

$$
\begin{aligned}
B_{u} u_{0}(0)+B_{v} v_{0}(0) & =b(0) \\
B_{u} v_{0}^{\prime}(0)+a B_{v} u_{0}^{\prime}(0) & =-b^{\prime}(0)+B_{u} q_{1}(0,0)+B_{v} q_{2}(0,0) .
\end{aligned}
$$

We denote

$$
\begin{aligned}
U^{\epsilon}=\left(\begin{array}{c}
u^{\epsilon} \\
v^{\epsilon}
\end{array}\right) & A=\left(\begin{array}{cc}
0 & I_{n} \\
a I_{n} & 0
\end{array}\right) & S=\left(\begin{array}{cc}
0 & 0 \\
F & -I_{n}
\end{array}\right) \\
Q=\left(\begin{array}{l}
q_{1} \\
q_{2}
\end{array}\right) & U_{0}=\left(\begin{array}{c}
u_{0} \\
v_{0}
\end{array}\right) & B=\left(B_{u}, B_{v}\right) .
\end{aligned}
$$

First for completeness, let us recall a definition on the $L^{2}$-well-posedness of the problem (1) from [7] as follows.

Definition 1.1. The IBVP (1) is stiffly well-posed if there exists a positive constant $K_{T}$ independent of $\epsilon$ such that

$$
\begin{aligned}
& \int_{0}^{T} \int_{0}^{\infty}\left|U^{\epsilon}(x, t)\right|^{2} d x d t+\int_{0}^{T}\left|U^{\epsilon}(0, t)\right|^{2} d t \\
& \quad \leq K_{T}\left(\int_{0}^{T}|b(t)|^{2} d t+\int_{0}^{\infty}\left|U_{0}(x)\right|^{2} d x+\int_{0}^{T} \int_{0}^{\infty}|Q(x, t)|^{2} d x d t\right)
\end{aligned}
$$

for all $U_{0} \in L^{2}\left(\mathbb{R}^{+}\right), b \in L^{2}\left(\mathbb{R}^{+}\right)$and $Q \in L^{2}\left(\mathbb{R}^{+} \times[0, T)\right)$ with $\mathbb{R}^{+}=\{x>0\}$.

We shall denote by $\|\cdot\|_{s}$ the classical $H^{s}$ norm, by $O(1)$ some absolute constants independent of $\epsilon, t, b(t), U_{0}(x)$ and $Q(x, t)$, and by $C_{0}$ some absolute constants depending only on $U_{0}(0), U_{0}^{\prime}(0)$ and $Q(0,0)$. Our main results are as follows.

Theorem 1.1. (IBVP, $\mathbf{n}=1$.) For the scalar case $n=1$, let $f(u)=\lambda u$, $\lambda \in R$, assume that the constant a satisfies the sub-characteristic condition

$$
a \geq \lambda^{2}
$$

and the boundary condition satisfies the following stiff Kreiss condition (SKC):

$$
B_{v}=0 \quad \text { or } \quad \frac{B_{u}}{B_{v}} \notin\left[-\sqrt{a},-\frac{\lambda+|\lambda|}{2}\right] .
$$

Then the IBVP (1) is stiffly well-posed. It holds:

1. Assume (7),(8) and $b \in L^{2}\left(\mathbb{R}^{+}\right), U_{0} \in H^{1}\left(\mathbb{R}^{+}\right), Q \in H^{1}\left(\mathbb{R}^{+} \times \mathbb{R}^{+}\right)$satisfying the compatibility condition (5). Then there exists a unique solution $U=(u, v)$ to the IBVP of the equilibrium system (3) such that

$$
\int_{0}^{\infty} \int_{0}^{\infty}\left|U^{\epsilon}-U\right|^{2} e^{-2 \alpha t} d x d t \rightarrow 0
$$

as $\epsilon \rightarrow 0$ for any $\alpha>0$. 
2. If we further assume $b \in H^{2}\left(\mathbb{R}^{+}\right), U_{0} \in H^{2}\left(\mathbb{R}^{+}\right), Q \in H^{1}\left(\mathbb{R}^{+} \times \mathbb{R}^{+}\right)$ satisfying the conditions (5) and (6) then

$$
\begin{aligned}
\int_{0}^{\infty} \int_{0}^{\infty}\left|U^{\epsilon}-U\right|^{2} e^{-2 \alpha t} d x d t \\
\leq \quad O(1) \epsilon\left\|v_{0}-f\left(u_{0}\right)\right\|_{0}^{2}+O(1) \epsilon^{2}\left\|U_{0}\right\|_{2}^{2} \\
\\
+ \begin{cases}O(1) \epsilon^{2}\left(\|b\|_{2}^{2}+\|Q\|_{1}^{2}+C_{0}\right) & \text { if } \lambda>0 \\
O(1) \epsilon^{\frac{1}{2}}\left(\|b\|_{0}^{2}+\|Q\|_{1}^{2}+C_{0}\right) & \text { if } \lambda=0 \\
O(1) \epsilon\left(\|b\|_{0}^{2}+\|Q\|_{1}^{2}+C_{0}\right) & \text { if } \lambda<0\end{cases}
\end{aligned}
$$

3. There exist an initial layer

$$
U^{I L}=U^{I L}\left(x, \frac{t}{\epsilon}\right)
$$

and a boundary layer

$$
U^{B L}= \begin{cases}0 & \text { if } \lambda>0 \\ U^{B L}\left(\frac{x}{\epsilon}, t\right) & \text { if } \lambda<0 \\ U^{B L}\left(\frac{x}{\sqrt{\epsilon}}, t\right) & \text { if } \lambda=0\end{cases}
$$

with $u^{I L}=0, v^{B L}=0$ such that

$$
\begin{aligned}
\int_{0}^{\infty} \int_{0}^{\infty} \mid U^{\epsilon}-U & -U^{I L}-\left.U^{B L}\right|^{2} e^{-2 \alpha t} d x d t \\
\leq & O(1) \epsilon^{2}\left\|U_{0}\right\|_{2}^{2} \\
& + \begin{cases}O(1) \epsilon^{2}\left(\|b\|_{2}^{2}+\|Q\|_{1}^{2}+C_{0}\right) & \text { if } \lambda \neq 0 \\
O(1) \epsilon^{\frac{3}{2}}\|b\|_{2}^{2}+O(1) \epsilon\left(\|Q\|_{1}^{2}+C_{0}\right) & \text { if } \lambda=0\end{cases}
\end{aligned}
$$

Theorem 1.2. (IBVP, $\mathbf{n}>$ 1.) Assume that the constant a satisfies the following sub-characteristic condition

$$
a \geq \max _{1 \leq i \leq n} \lambda_{i}^{2}
$$

and the boundary condition satisfies the following stiff Kreiss condition

$$
\left|\operatorname{det}\left(B_{u} R+B_{v} R G(\xi)\right)\right| \geq C
$$


for some $C>0$ and all $\xi \in \mathbb{C}$ with $\operatorname{Re} \xi \geq 0$, where

$$
\begin{aligned}
& G(\xi)=\operatorname{diag}\left\{g_{1}(\xi), g_{2}(\xi), \ldots, g_{n}(\xi)\right\} \\
& g_{j}(\xi)=\frac{\lambda_{j}+\sqrt{\lambda_{j}^{2}+4 a \xi(1+\xi)}}{2(1+\xi)}
\end{aligned}
$$

then the IBVP (1) is stiffly well-posed. It holds:

1. Assume (11), (12) and $b \in L^{2}\left(\mathbb{R}^{+}\right), U_{0} \in H^{1}\left(\mathbb{R}^{+}\right), Q \in H^{1}\left(\mathbb{R}^{+} \times \mathbb{R}^{+}\right)$ satisfying the condition (5). Then there exists a unique solution $U=(u, v)$ to the IBVP of (3) such that

$$
\int_{0}^{\infty} \int_{0}^{\infty}\left|U^{\epsilon}-U\right|^{2} e^{-2 \alpha t} d x d t \rightarrow 0
$$

as $\epsilon \rightarrow 0$ for any $\alpha>0$.

2. If we further assume $b \in H^{2}\left(\mathbb{R}^{+}\right), U_{0} \in H^{2}\left(\mathbb{R}^{+}\right)$and $Q \in H^{2}\left(\mathbb{R}^{+} \times \mathbb{R}^{+}\right)$ satisfying (5) and (6), then

$$
\begin{array}{rl}
\int_{0}^{\infty} \int_{0}^{\infty}\left|U^{\epsilon}-U\right|^{2} e^{-2 \alpha t} & d x d t \\
\leq & O(1) \epsilon^{\frac{1}{2}}\left(\|b\|_{2}^{2}+\|Q\|_{1}^{2}+C_{0}\right) \\
& +O(1) \epsilon^{2}\left\|U_{0}\right\|_{2}^{2}+O(1) \epsilon\left\|v_{0}-f\left(u_{0}\right)\right\|_{0}^{2}
\end{array}
$$

3. There exist an initial layer $U^{I L}=U^{I L}\left(x, \frac{t}{\epsilon}\right)$ and a boundary layer $U^{B L}$ with $u^{I L}=0, v^{B L}=0$ such that

$$
\begin{aligned}
\int_{0}^{\infty} \int_{0}^{\infty} \mid U^{\epsilon}-U & -U^{I L}-\left.U^{B L}\right|^{2} e^{-2 \alpha t} d x d t \\
& \leq O(1) \epsilon\left(\|b\|_{2}^{2}+\|Q\|_{1}^{2}+C_{0}\right)+O(1) \epsilon^{2}\left\|U_{0}\right\|_{2}^{2}
\end{aligned}
$$

Remark. From the stiff Kreiss condition (12), we can easily derive the boundary condition for the equilibrium problem. Without loss of generality, we assume that $F$ is diagonal:

$$
F=\left(\begin{array}{ccc}
\Lambda_{+} & & \\
& 0 & \\
& & \Lambda_{-}
\end{array}\right),
$$

where $\Lambda_{+}=\operatorname{diag}\left\{\lambda_{1}, \cdots, \lambda_{p}\right\}, \Lambda_{-}=\operatorname{diag}\left\{\lambda_{p+q+1}, \cdots, \lambda_{p+q+r}\right\}$ with $\lambda_{i}>0$ $(1 \leq i \leq p), \lambda_{i}<0(p+q+1 \leq i \leq p+q+r)$ and $p+q+r=n, p, q, r \in N$, 
$p, q, r \geq 0$. Correspondingly we set

$$
B_{u}=\left(\begin{array}{ccc}
B_{u}^{+} & & \\
& B_{u}^{n} & \\
& & B_{u}^{-}
\end{array}\right) \quad B_{v}=\left(\begin{array}{ccc}
B_{v}^{+} & & \\
& B_{v}^{n} & \\
& & B_{v}^{-}
\end{array}\right)
$$

and $u=\left(u_{+}, u_{n}, u_{-}\right)^{T}, v=\left(v_{+}, v_{n}, v_{-}\right)^{T}, b=\left(b_{+}, b_{n}, b_{-}\right)^{T}$ with same block size of $F$. By setting $\xi=0$ in (12), we have

$$
\left|\operatorname{det}\left(\begin{array}{ccc}
B_{u}^{+}+B_{v}^{+} \Lambda_{+} & & \\
& B_{u}^{n} & \\
& & B_{u}^{-}
\end{array}\right)\right| \geq C>0 .
$$

Therefore, we have

$$
\left|\operatorname{det}\left(B_{u}^{+}+B_{v}^{+} \Lambda_{+}\right)\right| \geq C>0 .
$$

Then the IBVP of the equilibrium system is given by

$$
\left\{\begin{aligned}
\partial_{t} u+F \partial_{x} u & =q_{1}, \\
v & =F u, \\
\left(B_{u}^{+}+B_{v}^{+} \Lambda_{+}\right) u_{+}(0, t) & =b_{+}(t), \\
u_{(x, 0)} & =u_{0}(x) .
\end{aligned}\right.
$$

It is easy to see that the IBVP of the equilibrium system is well-posed.

Remark. In contrast with [7], here we have established some more general results which remove the restrictions of [7]. Moreover, we extend the results in [9] to the inhomogeneous systems.

The remainder of this paper is arranged as follows: In Section 2, we will decompose the oringinal problem into two partial problems and give the solution in the scalar case. In Section 3, we will prove the asymptotic convergence of the solution of the relaxation system towards the solution of the corresponding equilibrium problem in the scaler case. In Section 4, we will calculate the boundary layer and establish the convergence rate in this case. In Section 5, we will prove Theorem 1.2.

\section{Solution of the problem: the scalar case}

In this section, we decompose the oringinal problem into two ones by a change of variables and give the solution of the problem. We rewrite the problem (1) as follows:

$$
\left\{\begin{aligned}
\partial_{t} U^{\epsilon}+A \partial_{x} U^{\epsilon} & =\frac{1}{\epsilon} S U^{\epsilon}+Q \\
U^{\epsilon}(x, 0) & =U_{0}(x) \\
B U^{\epsilon}(0, t) & =b(t)
\end{aligned}\right.
$$


2.1. Decomposition of the problem. The first step we take is to make a change of variables

$$
w^{\epsilon}=u^{\epsilon}-\bar{u}, \quad z^{\epsilon}=v^{\epsilon}-\bar{v},
$$

where $\bar{u}, \bar{v} \in H^{3}\left(\mathbb{R}^{+} \times \mathbb{R}^{+}\right)$are to be specified satisfying the conditions

$$
\begin{aligned}
\bar{u}(0,0) & =u_{0}(0) & \bar{v}(0,0) & =v_{0}(0) \\
\partial_{x} \bar{u}(0,0) & =u_{0}^{\prime}(0) & \partial_{x} \bar{v}(0,0) & =v_{0}^{\prime}(0) \\
\partial_{t} \bar{u}(0,0) & =-v_{0}^{\prime}(0)+q_{1}(0,0) & \partial_{t} \bar{v}(0,0) & =-a u_{0}^{\prime}(0)+q_{2}(0,0) \\
\bar{v} & =\lambda \bar{u} . & &
\end{aligned}
$$

We use the notations

$$
\bar{U}=\left(\begin{array}{c}
\bar{u} \\
\bar{v}
\end{array}\right) \quad W^{\epsilon}=\left(\begin{array}{c}
w^{\epsilon} \\
z^{\epsilon}
\end{array}\right)
$$

and rewrite system (15) with the above change of variables as

$$
\left\{\begin{aligned}
\partial_{t} W^{\epsilon}+A \partial_{x} W^{\epsilon} & =\frac{1}{\epsilon} S W^{\epsilon}-\left(\partial_{t} \bar{U}+A \partial_{x} \bar{U}\right)+Q \\
W^{\epsilon}(x, 0) & =U_{0}(x)-\bar{U}(x, 0) \\
B W^{\epsilon}(0, t) & =b(t)-B \bar{U}(0, t) .
\end{aligned}\right.
$$

The above problem does not change the form of the problem (15). We still denote it by

$$
\left\{\begin{aligned}
\partial_{t} W^{\epsilon}+A \partial_{x} W^{\epsilon} & =\frac{1}{\epsilon} S W^{\epsilon}+N \\
W^{\epsilon}(x, 0) & =W_{0}(x) \\
B W^{\epsilon}(0, t) & =c(t)
\end{aligned}\right.
$$

where $N=\left(\begin{array}{c}n_{1} \\ n_{2}\end{array}\right)$ and

$$
\begin{aligned}
N & =Q-\left(\partial_{t} \bar{U}+A \partial_{x} \bar{U}\right) \\
W_{0}(x) & =U_{0}(x)-\bar{U}(x, 0) \\
c(t) & =b(t)-B \bar{U}(0, t) .
\end{aligned}
$$

The compatibility conditions (5) and (6) of the problem (1) are equivalent to that (21) admits the following compatibility conditions:

$$
\begin{aligned}
& W_{0}(0)=0 \quad c(0)=0 \\
& W_{0}^{\prime}(0)=0 \quad c^{\prime}(0)=0 \quad N(0,0)=0 .
\end{aligned}
$$

Remark. The precise construction of $(\bar{u}, \bar{v})$ in $(17)-(20)$ was based on the compatibility conditions (5) and (6) hold simultaneously. In the case that one only has the zero-th order compatibility condition (5) for the problem (1), we can choose the functions $(\bar{u}, \bar{v})$ satisfying (17) and (20) only, and the transformed problem (21) admits the zero-th order compatibility condition (22) as well. 
Now, we begin to deal with the problem (21). Note that with the compatibility condition (22), we can decompose problem (21) into the two problems

$$
\left\{\begin{aligned}
\partial_{t} W^{\epsilon}+A \partial_{x} W^{\epsilon} & =\frac{1}{\epsilon} S W^{\epsilon} \\
W^{\epsilon}(x, 0) & =W_{0}(x) \\
B W^{\epsilon}(0, t) & =c(t)
\end{aligned}\right.
$$

and

$$
\left\{\begin{aligned}
\partial_{t} W^{\epsilon}+A \partial_{x} W^{\epsilon} & =\frac{1}{\epsilon} S W^{\epsilon}+N \\
W^{\epsilon}(x, 0) & =0 \\
B W^{\epsilon}(0, t) & =0 .
\end{aligned}\right.
$$

If (21) admits the compatibility conditions (22) and (23), the compatibilty conditions of (24) and (25) up to order one are satisfied as well. The first problem (24) is a homogeneous one which has been studied in [7]. We only need to focus on the second one (25).

In the end of this subsection, we show the structure of $\bar{U}$. With Taylor's series, it is easy to give a $C^{\infty}\left(R^{2}\right)$ function $V(x, t)$ satisfying the condition (17)-(20), namely

$$
V(x, t)=\left(\begin{array}{c}
u_{0}(0) \\
v_{0}(0)
\end{array}\right)+\left(\begin{array}{c}
u_{0}^{\prime}(0) x+\left(q_{1}(0,0)-v_{0}^{\prime}(0)\right) t \\
v_{0}^{\prime}(0) x+\left(q_{2}(0,0)-a u_{0}^{\prime}(0)\right) t
\end{array}\right) .
$$

If we set

$$
\bar{U}(x, t)=\phi\left(\frac{x}{\delta}, \frac{t}{\delta}\right) V(x, t),
$$

where $\phi(x, t) \in C_{0}^{\infty}\left(R_{+}^{2}\right)$ is a truncation function satisfying

$$
\phi(x, t)= \begin{cases}1 & 0 \leq x, t \leq \frac{1}{2} \\ 0 & x, t \geq 1\end{cases}
$$

then it is easy to verify that

$$
\|\bar{U}\|_{s}^{2} \leq O(1) \delta^{2-2 s}\left(\left|U_{0}(0)\right|^{2}+\left|U_{0}^{\prime}(0)\right|^{2}+b^{2}(0)+b^{\prime 2}(0)\right)
$$

By setting $\delta=1$, we get

$$
\|\bar{U}\|_{s}^{2} \leq C_{0}
$$

2.2. Solution by the Laplace transform. In this subsection, we study the problem (25). We denote the Laplace transform by

$$
\tilde{W}^{\epsilon}(x, \xi)=\mathcal{L} W^{\epsilon}=\int_{0}^{\infty} e^{-\xi t} W^{\epsilon}(x, t) d t, \quad \operatorname{Re} \xi \geq 0 .
$$


Therefore, the problem (25) becomes

$$
\left\{\begin{aligned}
\partial_{x} \tilde{W}^{\epsilon} & =\frac{1}{\epsilon} M(\epsilon \xi) \tilde{W}^{\epsilon}+A^{-1} \tilde{N}(x, \xi) \\
B \tilde{W}^{\epsilon}(0, \xi) & =0
\end{aligned}\right.
$$

where

$$
M(\xi)=A^{-1}(S-\xi I) .
$$

The general solution $\tilde{W}^{\epsilon}(x, \xi)$ of the equation in $(27)$ can be represented as

$$
\tilde{W}^{\epsilon}(x, \xi)=e^{M(\epsilon \xi) \frac{x}{\epsilon}}\left(\tilde{W}^{\epsilon}(0, \xi)+\int_{0}^{x} e^{-M(\epsilon \xi) \frac{y}{\epsilon}} A^{-1} \tilde{N}(y, \xi) d y\right)
$$

where

$$
e^{M(\xi) x}=e^{\mu_{+} x} \Phi_{+}(\xi)+e^{\mu_{-} x} \Phi_{-}(\xi)
$$

with

$$
\mu_{ \pm}=\frac{\lambda \pm \sqrt{\lambda^{2}+4 a \xi(1+\xi)}}{2 a}, \quad k(\xi)=\frac{a \mu_{-}(\xi)}{1+\xi}, \quad g(\xi)=\frac{a \mu_{+}(\xi)}{1+\xi}
$$

and

$$
\begin{aligned}
& \Phi_{+}(\xi)=\frac{1}{g(\xi)-k(\xi)}\left(\begin{array}{c}
1 \\
k(\xi)
\end{array}\right)(g(\xi),-1) \\
& \Phi_{-}(\xi)=\frac{1}{g(\xi)-k(\xi)}\left(\begin{array}{c}
1 \\
g(\xi)
\end{array}\right)\left(\begin{array}{ll}
-k(\xi), & 1
\end{array}\right) .
\end{aligned}
$$

Therefore $\tilde{W}^{\epsilon}$ can be rewritten as

$$
\begin{aligned}
\tilde{W}^{\epsilon}(x, \xi) & =e^{\mu_{+}(\epsilon \xi) \frac{x}{\epsilon}} \Phi_{+}(\epsilon \xi)\left(\tilde{W}^{\epsilon}(0, \xi)+\int_{0}^{x} e^{-\mu_{+}(\epsilon \xi) \frac{y}{\epsilon}} A^{-1} \tilde{N}(y, \xi) d y\right) \\
& +e^{\mu_{-}(\epsilon \xi) \frac{x}{\epsilon}} \Phi_{-}(\epsilon \xi)\left(\tilde{W}^{\epsilon}(0, \xi)+\int_{0}^{x} e^{-\mu_{-}(\epsilon \xi) \frac{y}{\epsilon}} A^{-1} \tilde{N}(y, \xi) d y\right) .
\end{aligned}
$$

The boundary value $\tilde{W}^{\epsilon}(0, \xi)$ remains to be determined. From the boundary condition

$$
B \tilde{W}^{\epsilon}(0, \xi)=0
$$

of the problem (25) and a natural boundary condition

$$
\Phi_{+}(\epsilon \xi)\left(\tilde{W}^{\epsilon}(0, \xi)+\int_{0}^{\infty} e^{-\mu_{+}(\epsilon \xi) \frac{y}{\epsilon}} A^{-1} \tilde{N}(y, \xi) d y\right)=0
$$

we have

$$
\tilde{W}^{\epsilon}(0, \xi)=\frac{\tilde{r}^{\epsilon}(\xi)}{B_{u}+B_{v} g(\epsilon \xi)}\left(\begin{array}{c}
B_{v} \\
-B_{u}
\end{array}\right),
$$


where

$$
\tilde{r}^{\epsilon}(\xi)=\int_{0}^{\infty} e^{-\mu_{+}(\epsilon \xi) \frac{y}{\epsilon}}\left(\tilde{n}_{1}(y, \xi)-\frac{1}{a} g(\epsilon \xi) \tilde{n}_{2}(y, \xi)\right) d y .
$$

The solution of problem (25) is given by

$$
\begin{aligned}
\tilde{W}^{\epsilon}(x, \xi) & =\frac{1}{g(\epsilon \xi)-k(\epsilon \xi)} \\
\times & {\left[\left(\begin{array}{c}
1 \\
k(\epsilon \xi)
\end{array}\right) \int_{x}^{\infty} e^{\mu_{+}(\epsilon \xi) \frac{(x-y)}{\epsilon}}\left(\tilde{n}_{1}(y, \xi)-\frac{1}{a} g(\epsilon \xi) \tilde{n}_{2}(y, \xi)\right) d y\right.} \\
+ & \left(\begin{array}{c}
1 \\
g(\epsilon \xi)
\end{array}\right) \int_{0}^{x} e^{\mu_{-}(\epsilon \xi) \frac{(x-y)}{\epsilon}}\left(\tilde{n}_{1}(y, \xi)-\frac{1}{a} k(\epsilon \xi) \tilde{n}_{2}(y, \xi)\right) d y \\
& \left.-\frac{B_{u}+B_{v} k(\epsilon \xi)}{B_{u}+B_{v} g(\epsilon \xi)} \tilde{r}^{\epsilon}(\xi) e^{\mu_{-}(\epsilon \xi) \frac{x}{\epsilon}}\left(\begin{array}{c}
1 \\
g(\epsilon \xi)
\end{array}\right)\right] .
\end{aligned}
$$

We denote by $\tilde{W}_{I}^{\epsilon}, \tilde{W}_{\mathbb{I I}}^{\epsilon}$ and $\tilde{W}_{\text {III }}^{\epsilon}$ the three items on the right side of the above equality respectively.

Remark. Since the uniform Lopatinski condition is just a consequence of the stiff Kreiss condition, the stiff well-posedness is easily established. We shall only focus on the asymptotic convergence and the boundary layer.

\section{Asymptotic convergence: the scalar case}

In this subsection, we show the convergence of $W^{\epsilon}$ towards the solution of the corresponding equilibrium system. It is easy to verify the following relations:

$$
\begin{aligned}
\left|\frac{\epsilon}{R e \mu_{+}(\epsilon \xi)}\right|=\left\{\begin{array}{ll}
O(1) \epsilon & \lambda>0 \\
O(1) \sqrt{\epsilon} & \lambda=0 \\
O(1) & \lambda<0
\end{array},\left|\frac{\epsilon}{\mu_{+}(\epsilon \xi)}\right|= \begin{cases}O(1) \epsilon & \lambda>0 \\
O(1)\left(\frac{\epsilon}{|\xi|}\right)^{\frac{1}{2}} & \lambda=0 \\
O(1)|\xi|^{-1} & \lambda<0\end{cases} \right. \\
\left|\frac{\epsilon}{R e \mu_{-}(\epsilon \xi)}\right|=\left\{\begin{array}{ll}
O(1) \epsilon & \lambda<0 \\
O(1) \sqrt{\epsilon} & \lambda=0 \\
O(1) & \lambda>0
\end{array},\left|\frac{\epsilon}{\mu_{-}(\epsilon \xi)}\right|= \begin{cases}O(1) \epsilon & \lambda<0 \\
O(1)\left(\frac{\epsilon}{|\xi|}\right)^{\frac{1}{2}} & \lambda=0 \\
O(1)|\xi|^{-1} & \lambda>0\end{cases} \right. \\
|k(\epsilon \xi)|=\left\{\begin{array}{ll}
O(1) \epsilon & \lambda>0 \\
O(1) \sqrt{\epsilon} & \lambda=0 \\
O(1) & \lambda<0
\end{array}, \quad|g(\epsilon \xi)|= \begin{cases}O(1) \epsilon & \lambda<0 \\
O(1) \sqrt{\epsilon} & \lambda=0 \\
O(1) & \lambda>0\end{cases} \right.
\end{aligned}
$$

as well as

$$
\left|\frac{1}{g(\epsilon \xi)-k(\epsilon \xi)}\right|= \begin{cases}O(1) & \lambda \neq 0 \\ O(1) \epsilon^{-1} & \lambda=0\end{cases}
$$


and

$$
\left|e^{z}-1\right| \leq O(1)|z| \quad \text { for } \operatorname{Re} z<0 .
$$

In the following discussion, we shall always set $\xi=\alpha+i \beta(\alpha, \beta \in R)$.

First, we deal with $W_{\text {III }}^{\epsilon}$. Thanks to the stiff Kreiss condition (8), we have

$$
0<C_{1} \leq\left|B_{u}+B_{v} g(\xi)\right| \leq C_{2}<\infty
$$

for all $\xi$ with $R e \xi \geq 0$. On the other hand, we have the following simple estimate on $\tilde{r}^{\epsilon}(\xi)$ :

$$
\begin{aligned}
\left|\tilde{r}^{\epsilon}(\xi)\right|^{2} & \leq\left|\int_{0}^{\infty} e^{-\mu_{+}(\epsilon \xi) \frac{y}{\epsilon}}\left(\tilde{n}_{1}(y, \xi)-\frac{1}{a} g(\epsilon \xi) \tilde{n}_{2}(y, \xi)\right) d y\right|^{2} \\
& \leq \int_{0}^{\infty}\left|e^{-\mu_{+}(\epsilon \xi) \frac{y}{\epsilon}}\right|^{2} d y \int_{0}^{\infty}\left|\tilde{n}_{1}(y, \xi)-\frac{1}{a} g(\epsilon \xi) \tilde{n}_{2}(y, \xi)\right|^{2} d y \\
& \leq O(1) \frac{\epsilon}{R e \mu_{+}(\epsilon \xi)} \int_{0}^{\infty}|\tilde{N}(y, \xi)|^{2} d y .
\end{aligned}
$$

When $\lambda \neq 0$, noticing (29), (32) and (34), we have

$$
\begin{aligned}
\int_{0}^{\infty} \int_{0}^{\infty} \mid & \left.W_{\text {III }}^{\epsilon}(x, t)\right|^{2} e^{-2 \alpha t} d x d t \\
& =\int_{0}^{\infty}\left(\int_{-\infty}^{\infty}\left|\tilde{W}_{\text {III }}^{\epsilon}(x, \xi)\right|^{2} d \beta\right) d x \\
& \leq O(1)-\frac{\epsilon}{R e \mu_{+}(\epsilon \xi)} \cdot \frac{\epsilon}{R e \mu_{-}(\epsilon \xi)} \int_{0}^{\infty} \int_{-\infty}^{\infty}|\tilde{N}(x, \xi)|^{2} d \beta d x \\
& \leq O(1) \epsilon \int_{0}^{\infty} \int_{0}^{\infty}|N(x, t)|^{2} d x d t
\end{aligned}
$$

When $\lambda=0$, due to (32), we can't derive a estimate similar to $(36)$, if $N(x, t) \in$ $L^{2}\left(\mathbb{R}^{+} \times \mathbb{R}^{+}\right)$. Therefore, if $N(x, t) \in H^{1}\left(\mathbb{R}^{+} \times \mathbb{R}^{+}\right)$, by integration by parts we have

$$
\tilde{W}_{\text {III }}^{\epsilon}=\left(\begin{array}{c}
1 \\
g(\epsilon \xi)
\end{array}\right) \frac{e^{\mu_{-}(\epsilon \xi) \frac{x}{\epsilon}}\left(B_{u}+B_{v} k(\epsilon \xi)\right)}{2 \xi\left(B_{u}+B_{v} k(\epsilon \xi)\right)}\left(\tilde{n}_{1}(0, \xi)-\frac{1}{a} g(\epsilon \xi) \tilde{n}_{2}(0, \xi)-\tilde{s}^{\epsilon}(\xi)\right)
$$

where

$$
\tilde{s}^{\epsilon}(\xi)=\int_{0}^{\infty} e^{-\mu_{+}(\epsilon \xi) \frac{y}{\epsilon}}\left(\partial_{y} \tilde{n}_{1}(y, \xi)-\frac{1}{a} g(\epsilon \xi) \partial_{y} \tilde{n}_{2}(y, \xi)\right) d y .
$$

Similar to (35), we can prove

$$
\int_{0}^{\infty} \int_{0}^{\infty} e^{-2 \alpha t}\left|W_{\text {III }}^{\epsilon}\right|^{2} d x d t \leq O(1) \epsilon\|N\|_{1}^{2}
$$

Next, we establish the covergence of $W_{I}^{\epsilon}+W_{\text {II }}^{\epsilon}$ towards the solution of the corresponding equilibrium system. For this we consider two cases. 
3.1. The case of non-characteristic boundary. When $\lambda>0$, the corresponding problem of the equilibrium system is as follows:

$$
\left\{\begin{aligned}
\partial_{t} w+\lambda \partial_{x} w & =n_{1} \\
z & =\lambda w \\
w(x, 0) & =0 \\
w(0, t) & =0
\end{aligned}\right.
$$

By a simple calculation, we get the solution

$$
\tilde{W}=\left(\begin{array}{c}
\frac{1}{\lambda} \\
1
\end{array}\right) \int_{0}^{x} e^{-\frac{(x-y)}{\lambda}} \tilde{n}_{1}(u, \xi) d y
$$

By using (31) and (32), we have

$$
\int_{-\infty}^{\infty} \int_{0}^{\infty}\left|\tilde{W}_{I}^{\epsilon}\right|^{2} d x d \beta \leq O(1) \int_{-\infty}^{\infty} \int_{0}^{\infty}\left(\int_{x}^{\infty} e^{R e \mu_{+}(\epsilon \xi) \frac{(x-y)}{\epsilon}}|\tilde{N}(y, \xi)| d y\right)^{2} d x d \beta
$$

Denote the item on the right side of the above inequality by $I$. By integration by parts, we have for $I$

$$
\begin{aligned}
I= & \frac{\epsilon}{2 \operatorname{Re} \mu_{+}(\epsilon \xi)}\left(\left.\int_{-\infty}^{\infty}\left(\int_{x}^{\infty} e^{R e \mu_{+}(\epsilon \xi) \frac{(x-y)}{\epsilon}}|\tilde{N}(y, \xi)| d y\right)^{2}\right|_{x=0} ^{\infty} d \beta\right. \\
& \left.+2 \int_{-\infty}^{\infty} \int_{0}^{\infty}|\tilde{N}(x, \xi)|\left(\int_{x}^{\infty} e^{R e \mu_{+}(\epsilon \xi) \frac{(x-y)}{\epsilon}}|\tilde{N}(y, \xi)| d y\right) d x d \beta\right) \\
\leq & \int_{-\infty}^{\infty} \int_{0}^{\infty}\left(\frac{\epsilon}{\operatorname{Re} \mu_{+}(\epsilon \xi)}|\tilde{N}(x, \xi)|\right) \\
& \times\left(\frac{1}{2} \int_{x}^{\infty} e^{R e \mu_{+}(\epsilon \xi) \frac{(x-y)}{\epsilon}}|\tilde{N}(y, \xi)| d y\right) d x d \beta \\
\leq & 2\left(\frac{\epsilon}{R e \mu_{+}(\epsilon \xi)}\right)^{2} \int_{-\infty}^{\infty} \int_{0}^{\infty}|\tilde{N}(x, \xi)|^{2} d x d \beta+\frac{1}{2} I
\end{aligned}
$$

Therefore by using (29), we have

$$
\begin{aligned}
\int_{-\infty}^{\infty} \int_{0}^{\infty}\left|\tilde{W}_{I}^{\epsilon}\right|^{2} d x d \beta & \leq O(1) I \\
& \leq O(1) \epsilon^{2} \int_{-\infty}^{\infty} \int_{0}^{\infty}|\tilde{N}(x, \xi)|^{2} d x d \beta \\
& \leq O(1) \epsilon^{2}\|N\|_{0}^{2}
\end{aligned}
$$


When considering $\tilde{W}_{\text {II }}^{\epsilon}$, we need to use the following estimates:

$$
\begin{aligned}
\left|\frac{1}{g(\epsilon \xi)-k(\epsilon \xi)}-\frac{1}{\lambda}\right| & =O(1) \epsilon & \left|\frac{k(\epsilon \xi)}{g(\epsilon \xi)-k(\epsilon \xi)}\right| & =O(1) \epsilon \\
\left|\frac{\mu_{-}(\epsilon \xi)}{\epsilon}+\frac{\xi}{\lambda}\right| & =O(1)|\xi| \epsilon & \left|\frac{\epsilon}{\mu_{-}(\epsilon \xi)}+\frac{\lambda}{\xi}\right| & =O(1) \epsilon .
\end{aligned}
$$

We write $\tilde{W}_{I I}^{\epsilon}-\tilde{W}$ as

$$
\begin{aligned}
\tilde{W}_{I I}^{\epsilon}-\tilde{W} & =\left(\begin{array}{c}
\frac{1}{g(\epsilon \xi)-k(\epsilon \xi)}-\frac{1}{\lambda} \\
\frac{k(\epsilon \xi)}{g(\epsilon \xi)-k(\epsilon \xi)}
\end{array}\right) \int_{0}^{x} e^{\mu_{-}(\epsilon \xi) \frac{(x-y)}{\epsilon}}\left(\tilde{n}_{1}-\frac{1}{a} k(\epsilon \xi) \tilde{n}_{2}(y, \xi)\right) d y \\
& -\left(\begin{array}{c}
\frac{1}{\lambda} \\
1
\end{array}\right) \int_{0}^{x} e^{\mu_{-}(\epsilon \xi) \frac{(x-y)}{\epsilon}} \frac{1}{a} k(\epsilon \xi) \tilde{n}_{2}(y, \xi) d y \\
& +\left(\begin{array}{c}
\frac{1}{\lambda} \\
1
\end{array}\right) \int_{0}^{x}\left(e^{\mu_{-}(\epsilon \xi) \frac{(x-y)}{\epsilon}}-e^{-\xi(x-y) / \lambda}\right) \tilde{n}_{1}(y, \xi) d y .
\end{aligned}
$$

Denote the three items on the right side of the above equality by $I$, II and III respectively. Similar to the proof of (36), we can prove the following estimates for $I$ and $I I$ :

$$
\begin{aligned}
& \int_{-\infty}^{\infty} \int_{0}^{\infty}|I|^{2} d x d \beta \leq O(1) \epsilon^{2}\|N\|_{0}^{2} \\
& \int_{-\infty}^{\infty} \int_{0}^{\infty}|I|^{2} d x d \beta \leq O(1) \epsilon^{2}\|N\|_{0}^{2}
\end{aligned}
$$

If $N(x, t) \in H^{1}\left(\mathbb{R}^{+} \times \mathbb{R}^{+}\right)$, we have for $\mathbb{I I}$ by integration by parts

$$
\begin{aligned}
|I I I| \leq & \left|\frac{\epsilon}{\mu_{-}(\epsilon \xi)}+\frac{\lambda}{\xi}\right| \cdot\left|\tilde{n}_{1}(x, \xi)\right|+\left|\frac{\epsilon}{\mu_{-}(\epsilon \xi)} e^{\mu_{-}(\epsilon \xi) \frac{x}{\epsilon}}+\frac{\lambda}{\xi} e^{-\xi \frac{x}{\lambda}}\right| \cdot\left|\tilde{n}_{1}(0, \xi)\right| \\
& +\left|\frac{\epsilon}{\mu_{-}(\epsilon \xi)} e^{\mu_{-}(\epsilon \xi) \frac{x}{\epsilon}}+\frac{\lambda}{\xi} e^{-\xi x / \lambda}\right| \cdot\left|\int_{0}^{x} e^{\mu_{-}(\epsilon \xi) \frac{y}{\epsilon}} \partial_{y} \tilde{n}_{1}(y, \xi) d y\right| \\
& +\left|\frac{\lambda}{\xi} e^{-\xi x / \lambda}\right| \cdot\left|\int_{0}^{x}\left(e^{\mu_{-}(\epsilon \xi) \frac{y}{\epsilon}}-e^{\xi \frac{y}{\lambda}} y\right) \partial_{y} \tilde{n}_{1}(y, \xi) d y\right| \\
\leq & O(1) \epsilon\left[\left|\tilde{n}_{1}(x, \xi)\right|+(1+x) e^{R e \mu_{-}(\epsilon \xi) \frac{x}{\epsilon}}\left|\tilde{n}_{1}(0, \xi)\right|\right. \\
& +(1+x) \int_{0}^{x} e^{R e \mu_{-}(\epsilon \xi) \frac{(x-y)}{\epsilon}}\left|\partial_{y} \tilde{n}_{1}(y, \xi)\right| d y \\
& \left.+x \int_{0}^{x} e^{-\operatorname{Re} \xi \frac{(x-y)}{\lambda}}\left|\partial_{y} \tilde{n}_{1}(y, \xi)\right| d y\right] .
\end{aligned}
$$


Therefore, we have

$$
\begin{aligned}
\int_{\infty}^{\infty} \int_{0}^{\infty}|\operatorname{III}|^{2} d x d \beta \leq & O(1) \epsilon^{2}\left[\int_{-\infty}^{\infty} \int_{0}^{\infty}\left|\tilde{n}_{1}(x, \xi)\right|^{2} d x d \beta+\int_{-\infty}^{\infty}\left|\tilde{n}_{1}(0, \xi)\right|^{2} d \beta\right. \\
& +\int_{-\infty}^{\infty} \int_{0}^{\infty}\left(\int_{0}^{x} e^{\operatorname{Re} \mu_{-}(\epsilon \xi) \frac{(x-y)}{\epsilon}}\left|\partial_{y} \tilde{n}_{1}(y, \xi)\right| d y\right)^{2} d x d \beta \\
& +\int_{-\infty}^{\infty} \int_{0}^{\infty}\left(\int_{0}^{x} x e^{\operatorname{Re} \mu_{-}(\epsilon \xi) \frac{(x-y)}{\epsilon}}\left|\partial_{y} \tilde{n}_{1}(y, \xi)\right| d y\right)^{2} d x d \beta \\
& \left.+\int_{-\infty}^{\infty} \int_{0}^{\infty}\left(\int_{0}^{x} e^{-\operatorname{Re} \frac{(x-y)}{\lambda}}\left|\partial_{y} \tilde{n}_{1}(y, \xi)\right| d y\right)^{2} d x d \beta\right]
\end{aligned}
$$

Denote the four items on the right side of the above inequality by $I_{1}, I_{2}, I_{3}$ and $I_{4}$ respectively. By using the trace theorem we have for $I_{1}$

$$
I_{1} \leq O(1)\|N\|_{1}^{2}
$$

Similar to the proof of (38), we can prove for $I_{2}$ the estimate

$$
I_{2} \leq O(1)\|N\|_{1}^{2}
$$

Considering $I_{3}$, by integration by parts we get

$$
\begin{aligned}
I_{3}= & \frac{\epsilon}{2 R e \mu_{-}(\epsilon \xi)} \int_{-\infty}^{\infty}\left[\left.\left(x \int_{0}^{x} e^{R e \mu_{-}(\epsilon \xi) \frac{(x-y)}{\epsilon}}\left|\partial_{y} \tilde{n}_{1}(y, \xi)\right| d y\right)^{2}\right|_{x=0} ^{\infty}\right. \\
& -2 \int_{0}^{\infty} x e^{2 R e \mu_{-}(\epsilon \xi) \frac{x}{\epsilon}}\left(\int_{0}^{x} e^{-R e \mu_{-} \frac{y}{\epsilon}}\left|\partial_{y} \tilde{n}_{1}(y, \xi)\right| d y\right)^{2} d x \\
& \left.-2 \int_{0}^{\infty} x e^{R e \mu_{-}(\epsilon \xi) \frac{x}{\epsilon}}\left(\int_{0}^{x} e^{-R e \mu_{-} \frac{y}{\epsilon}}\left|\partial_{y} \tilde{n}_{1}(y, \xi)\right| d y\right)\left|\partial_{x} \tilde{n}_{1}(x, \xi)\right| d x\right] d \beta \\
\leq & \int_{-\infty}^{\infty} \int_{0}^{\infty}\left(-\frac{3 \epsilon}{R e \mu_{-}(\epsilon \xi)} \int_{0}^{x} e^{R e \mu_{-}(\epsilon \xi) \frac{(x-y)}{\epsilon}}\left|\partial_{y} \tilde{n}_{1}(y, \xi)\right| d y\right. \\
& \left.\times \frac{1}{3} \int_{0}^{x} x e^{R e \mu_{-}(\epsilon \xi) \frac{(x-y)}{\epsilon}}\left|\partial_{y} \tilde{n}_{1}(y, \xi)\right| d y\right) d x d \beta \\
& +\int_{-\infty}^{\infty} \int_{0}^{\infty}\left(-\frac{3 \epsilon}{R e \mu_{-}(\epsilon \xi)}\left|\partial_{x} \tilde{n}_{1}(x, \xi)\right|\right. \\
& \left.\times \frac{1}{3} \int_{0}^{x} x e^{R e \mu_{-}(\epsilon \xi) \frac{(x-y)}{\epsilon}}\left|\partial_{y} \tilde{n}_{1}(y, \xi)\right| d y\right) d x d \beta \\
\leq & 18\left(\frac{\epsilon}{R e \mu_{-}(\epsilon \xi)}\right)^{2} I_{2}+\frac{2}{9} I_{3} \\
& +18\left(\frac{\epsilon}{R e \mu_{-}(\epsilon \xi)}\right)^{2} \int_{-\infty}^{\infty} \int_{0}^{\infty}\left|\partial_{y} \tilde{n}_{1}(x, \xi)\right|^{2} d x d \beta+\frac{2}{9} I_{3}
\end{aligned}
$$


Therefore, with the estimate of $I_{2}$, we get

$$
I_{3} \leq O(1)\|N\|_{1}^{2}
$$

Similar to (42), we can establish the estimate

$$
I_{4} \leq O(1)\|N\|_{1}^{2}
$$

Combining all the estimates of $I_{k}(k=1,2,3,4)$ in the above, we have

$$
\int_{-\infty}^{\infty} \int_{0}^{\infty}\left|\tilde{W}_{I I}^{\epsilon}-\tilde{W}\right|^{2} d x d \beta \leq O(1) \epsilon^{2}\|N\|_{1}^{2}
$$

Therefore, we get the asymptotic convergence of $W_{I}^{\epsilon}+W_{I I}^{\epsilon}$ towards the solution $W$ of the corresponding problem of the equilibrium system via

$$
\int_{0}^{\infty} \int_{0}^{\infty} e^{-2 \alpha t}\left|W_{I}^{\epsilon}+W_{I I}^{\epsilon}-W\right|^{2} d x d \beta \leq O(1) \epsilon^{2}\|N\|_{1}^{2} \rightarrow 0
$$

as $\epsilon \rightarrow 0$.

When $\lambda<0$, the corresponding problem of the equilibrium system is given by

$$
\left\{\begin{aligned}
\partial_{t} w+\lambda \partial_{x} w & =n_{1} \\
z & =\lambda w \\
w(x, 0) & =0
\end{aligned}\right.
$$

The solution of the above problem is

$$
\tilde{W}=\left(\begin{array}{c}
-\frac{1}{\lambda} \\
1
\end{array}\right) \int_{x}^{\infty} e^{-\xi \frac{(x-y)}{\lambda}} \tilde{n}_{1}(u, \xi) d y .
$$

Similar to the case of $\lambda>0$, we can prove that the following estimates are true in the case of $\lambda<0$ :

$$
\int_{-\infty}^{\infty} \int_{0}^{\infty}\left|\tilde{W}_{I}-\tilde{W}\right|^{2} d x d \beta \leq O(1) \epsilon^{2}\|N\|_{1}^{2}
$$

and

$$
\int_{-\infty}^{\infty} \int_{0}^{\infty}\left|\tilde{W}_{I I}\right|^{2} d x d t \leq O(1) \epsilon^{2}\|N\|_{0}^{2}
$$

Here we omit the details of the derivation of the above estimates. Therefore, we can prove that (44) is also true in this case. 
3.2. The case of uniform characteristic boundary. When $\lambda=0$, the corresponding problem of the equilibrium system is given by

$$
\left\{\begin{aligned}
\partial_{t} w & =n_{1} \\
z & =0 \\
w(x, 0) & =0 .
\end{aligned}\right.
$$

The solution of the above problem is

$$
\tilde{W}=\left(\begin{array}{c}
\frac{\tilde{n}_{1}}{\xi} \\
0
\end{array}\right) .
$$

If $N(x, t) \in H^{1}\left(\mathbb{R}^{+} \times \mathbb{R}^{+}\right)$, then by integration by parts we have

$$
\begin{aligned}
\tilde{W}_{I}^{\epsilon}+ & \tilde{W}_{\text {II }}^{\epsilon}-\tilde{W} \\
= & \frac{1+\epsilon \xi}{2 \xi}\left[-\left(\begin{array}{c}
1 \\
g(\epsilon \xi)
\end{array}\right) e^{\mu_{-}(\epsilon \xi) \frac{x}{\epsilon}}\left(\tilde{n}_{1}(0, \xi)-\frac{1}{a} k(\epsilon \xi) \tilde{n}_{1}(0, \xi)\right)\right. \\
& +\left(\begin{array}{c}
1 \\
k(\epsilon \xi)
\end{array}\right) \int_{x}^{\infty} e^{\mu_{+}(\epsilon \xi) \frac{(x-y)}{\epsilon}}\left(\partial_{y} \tilde{n}_{1}(y, \xi)-\frac{1}{a} g(\epsilon \xi) \partial_{y} \tilde{n}_{1}(y, \xi)\right) d y \\
& \left.-\left(\begin{array}{c}
1 \\
g(\epsilon \xi)
\end{array}\right) \int_{0}^{x} e^{\mu_{-}(\epsilon \xi) \frac{(x-y)}{\epsilon}}\left(\partial_{y} \tilde{n}_{1}(y, \xi)-\frac{1}{a} k(\epsilon \xi) \partial_{y} \tilde{n}_{1}(y, \xi)\right) d y\right] \\
& +\left(\begin{array}{c}
0 \\
\frac{a^{2} \epsilon \tilde{n}_{2}(x, \xi)}{1+\epsilon \xi}
\end{array}\right) .
\end{aligned}
$$

Denote the four items on the right side of the above equality by $I_{1}, I_{2}, I_{3}$ and $I_{4}$ respectively. By direct calculation and using (31), we have for $I_{1}$ and $I_{4}$ the estimates

$$
\begin{aligned}
& \int_{-\infty}^{\infty} \int_{0}^{\infty}\left|I_{1}\right|^{2} d x d \beta \leq O(1) \epsilon^{\frac{1}{2}}\|N\|_{1}^{2} \\
& \int_{-\infty}^{\infty} \int_{0}^{\infty}\left|I_{4}\right|^{2} d x d \beta \leq O(1) \epsilon^{2}\|N\|_{0}^{2} .
\end{aligned}
$$

Similar to (39), we can establish the following estimates for $I_{2}$ and $I_{3}$ :

$$
\begin{aligned}
\int_{-\infty}^{\infty} \int_{0}^{\infty}\left|I_{2}\right|^{2} d x d \beta & \leq O(1)\left(\frac{\epsilon}{R e \mu_{+}(\epsilon \xi)}\right)^{2} \int_{-\infty}^{\infty} \int_{0}^{\infty}\left|\partial_{x} \tilde{N}(x, \xi)\right|^{2} d x d \beta \\
& \leq O(1) \epsilon\|N\|_{1}^{2}
\end{aligned}
$$

and

$$
\begin{aligned}
\int_{-\infty}^{\infty} \int_{0}^{\infty}\left|I_{3}\right|^{2} d x d \beta & \leq O(1)\left(\frac{\epsilon}{R e \mu_{-}(\epsilon \xi)}\right)^{2} \int_{-\infty}^{\infty} \int_{0}^{\infty}\left|\partial_{x} \tilde{N}(x, \xi)\right|^{2} d x d \beta \\
& \leq O(1) \epsilon\|N\|_{1}^{2}
\end{aligned}
$$


Therefore, we get the convergence of $W_{I}^{\epsilon}+W_{I I}^{\epsilon}$ towards $W$ according

$$
\int_{0}^{\infty} \int_{0}^{\infty} e^{-2 \alpha t}\left|W_{I}^{\epsilon}+W_{I I}^{\epsilon}-\tilde{W}\right|^{2} d x d t \leq O(1) \epsilon^{\frac{1}{2}}\|N\|_{1}^{2}
$$

Thus combining all the convergence results in this section, together with the results in [7], we have proved the asymptotic convergence and the estimate (9) for the convergence rate.

\section{Boundary layers and convergence rate: the scalar case}

In this section, we will study the boundary layer in the problem (25) and establish the convergence rate when the compatibility conditions (22) and (23) hold. For this we consider two cases.

4.1. The case of non-characteristic boundary. In the non-characteristic boundary case, namely $\lambda \neq 0$, we propose the following well-known expansion:

$$
\left\{\begin{aligned}
w^{\epsilon}(x, t) & =w(x, t)+w^{B L}(y, t)+O(1) \epsilon \\
z^{\epsilon}(x, t) & =z(x, t)+z^{B L}(y, t)+O(1) \epsilon
\end{aligned}\right.
$$

where $w^{B L}(y, t), z^{B L}(y, t)$ are the boundary layers decaying exponentially fast in $y=\frac{x}{\epsilon}$ when $y$ goes to infinity. By plugging (46) into (25) and matching the expansion, we get the boundary layer equations

$$
\left\{\begin{aligned}
\partial_{y} z^{B L} & =0 \\
a \partial_{y} w^{B L} & =\lambda w^{B L}-z^{B L}
\end{aligned}\right.
$$

We now specify the initial and boundary data in order to determine the boundary layer. When $\lambda>0$, we have

$$
\left\{\begin{aligned}
a \partial_{y} w^{B L} & =\lambda w^{B L}-z^{B L} \\
\partial_{y} z^{B L} & =0 \\
w^{B L}(0, t) & =0 \\
z^{B L}(0, t) & =0 .
\end{aligned}\right.
$$

It is easy to get $w^{B L}=z^{B L}=0$. No boundary layer develops in this case. Considering the convergence rate, noticing (30), we have the following estimates for $\tilde{r}^{\epsilon}(\xi)$ by integration by parts:

$$
\begin{aligned}
\left|\tilde{r}^{\epsilon}(\xi)\right|^{2}= & \left|\frac{\epsilon}{\mu_{+}(\epsilon \xi)}\right|^{2} \cdot \mid \int_{0}^{\infty} e^{-\mu_{+}(\epsilon \xi) \frac{y}{\epsilon}}\left(\partial_{y} \tilde{n}_{1}(y, \xi)-\frac{1}{a} g(\epsilon \xi) \partial_{y} \tilde{n}_{2}(y, \xi)\right) d y \\
& \quad+\tilde{n}_{1}(0, \xi)-\left.\frac{1}{a} g(\epsilon \xi) \tilde{n}_{2}(0, \xi)\right|^{2} \\
\leq & O(1) \epsilon^{2}\left(|\tilde{N}(0, \xi)|^{2}+\int_{0}^{\infty}\left|\partial_{y} \tilde{N}(y, \xi)\right|^{2} d y\right)
\end{aligned}
$$


Therefore, the above estimate yields

$$
\begin{aligned}
\int_{0}^{\infty} & \int_{0}^{\infty} e^{-2 \alpha t}\left|W_{\text {III }}(x, t)\right|^{2} d x d t \\
& =\int_{0}^{\infty} \int_{-\infty}^{\infty}\left|\tilde{W}_{\mathbb{I I I}}(\xi, x)\right|^{2} d \beta d x \\
& \leq O(1) \epsilon^{2}\left(\int_{-\infty}^{\infty}|\tilde{N}(0, \xi)|^{2} d \beta+\int_{0}^{\infty} \int_{-\infty}^{\infty}\left|\partial_{y} \tilde{N}(y, \xi)\right|^{2} d \beta d y\right) \\
& =O(1) \epsilon^{2}\left(\int_{0}^{\infty} e^{-2 \alpha t}|N(0, t)|^{2} d t+\int_{0}^{\infty} \int_{0}^{\infty} e^{-2 \alpha t}\left|\partial_{y} N(y, t)\right|^{2} d y d t\right) \\
& \leq O(1) \epsilon^{2}\|N(x, t)\|_{1}^{2} .
\end{aligned}
$$

When $\lambda<0$, the problem of the boundary is as follows:

$$
\left\{\begin{aligned}
a \partial_{y} w^{B L} & =\lambda w^{B L}-z^{B L} \\
\partial_{y} z^{B L} & =0 \\
B_{u} w^{B L}(0, t) & =-\left(B_{u}+\lambda B_{v}\right) w(0, t) \\
z^{B L}(0, t) & =0
\end{aligned}\right.
$$

Noticing (8), which implies $B_{u} \neq 0$ for $\lambda<0$, we give the boundary layers by

$$
\begin{aligned}
\tilde{w}^{B L}(x, \xi) & =\frac{B_{u}+\lambda B_{v}}{\lambda B_{u}} e^{\frac{\lambda x}{a \epsilon}} \int_{0}^{\infty} e^{\xi \frac{y}{\lambda}} \tilde{n}_{1}(y, \xi) d y \\
\tilde{z}^{B L} & =0 .
\end{aligned}
$$

To consider the convergence rate, we write

$$
\begin{aligned}
\tilde{w}_{\text {II }}^{\epsilon}-\tilde{w}^{B L} & \\
= & \frac{B_{u}+B_{v} k(\epsilon \xi)}{B_{u}+B_{v} g(\epsilon \xi)} \cdot \frac{g(\epsilon \xi) e^{\mu_{-}(\epsilon \xi) \frac{x}{\epsilon}}}{a(g(\epsilon \xi)-k(\epsilon \xi))} \int_{0}^{\infty} e^{-\mu_{+}(\epsilon \xi) \frac{y}{\epsilon}} \tilde{n}_{2}(y, \xi) d y \\
& +\left(-\frac{1}{g(\epsilon \xi)-k(\epsilon \xi)} \cdot \frac{B_{u}+B_{v} k(\epsilon \xi)}{B_{u}+B_{v} g(\epsilon \xi)}-\frac{B_{u}+\lambda B_{v}}{\lambda B_{u}}\right) e^{\mu_{-}(\epsilon \xi) \frac{x}{\epsilon}} \\
& \times \int_{0}^{\infty} e^{-\mu_{+}(\epsilon \xi) \frac{y}{\epsilon}} \tilde{n}_{1}(y, \xi) d y \\
& +\frac{B_{u}+\lambda B_{v}}{\lambda B_{u}} e^{\mu_{-}(\epsilon \xi) \frac{x}{\epsilon}} \int_{0}^{\infty}\left(e^{-\mu_{+}(\epsilon \xi) \frac{y}{\epsilon}}-e^{\xi \frac{y}{\lambda}}\right) \tilde{n}_{1}(y, \xi) d y \\
& +\frac{B_{u}+\lambda B_{v}}{\lambda B_{u}}\left(e^{\mu_{-}(\epsilon \xi) \frac{x}{\epsilon}}-e^{\frac{\lambda x}{a \epsilon}}\right) \int_{0}^{\infty} e^{\xi \frac{y}{\lambda}} \tilde{n}_{1}(y, \xi) d y .
\end{aligned}
$$


Denote the four items on the right hand side of the above inquality by $I_{1}, I_{2}, I_{3}$, and $I_{4}$ respectively. We estimate the four items separately. By using (29) - (32) and (34), similar to (36), we can prove for $I_{1}$

$$
\int_{0}^{\infty} \int_{-\infty}^{\infty}\left|I_{1}\right|^{2} d \beta d x \leq O(1) \epsilon^{3}\|N\|_{0}^{2}
$$

From (30) and (31), we have

$$
\left|-\frac{1}{g(\epsilon \xi)-k(\epsilon \xi)} \cdot \frac{B_{u}+B_{v} k(\epsilon \xi)}{B_{u}+B_{v} g(\epsilon \xi)}-\frac{B_{u}+\lambda B_{v}}{\lambda B_{u}}\right| \leq O(1) \epsilon .
$$

Thus, similar to (39), we have for $I_{2}$

$$
\int_{0}^{\infty} \int_{-\infty}^{\infty}\left|I_{2}\right|^{2} d \beta d x \leq O(1) \epsilon^{3}\|N\|_{0}^{2}
$$

To deal with $I_{3}$, the following estimate works:

$$
\left|\frac{\epsilon}{\mu_{+}(\epsilon \xi)}+\frac{\lambda}{\xi}\right| \leq O(1) \epsilon
$$

Together with (33) we have

$$
\left|\frac{\epsilon}{\mu_{+}(\epsilon \xi)} e^{-\mu_{+}(\epsilon \xi) \frac{y}{\epsilon}}+\frac{\lambda}{\xi} e^{\xi \frac{y}{\lambda}}\right| \leq O(1) \epsilon(1+y) e^{-2 \operatorname{Re} \mu_{+}(\epsilon \xi) \frac{y}{\epsilon}} .
$$

Therefore, by integration by parts, we have for $I_{3}$

$$
\begin{aligned}
\int_{0}^{\infty} \int_{-\infty}^{\infty}\left|I_{3}\right|^{2} d \beta d x \leq & O(1) \epsilon^{3}\left(\int_{-\infty}^{\infty}\left|\tilde{n}_{1}(0, \xi)\right|^{2} d \beta\right. \\
& \left.+\int_{-\infty}^{\infty}\left|\int_{0}^{\infty}(1+y) e^{-\mu_{+}(\epsilon \xi) \frac{y}{\epsilon}} \partial_{y} \tilde{n}_{1}(y, \xi) d y\right|^{2} d \beta\right) \\
\leq & O(1) \epsilon^{3}\|N\|_{1}^{2}
\end{aligned}
$$

According to the estimate

$$
\left|\frac{\mu_{-}(\epsilon \xi)}{\epsilon}-\frac{\lambda}{a \epsilon}\right| \leq O(1) \epsilon x|\xi|
$$

and by integration by parts, we have for $I_{4}$ 


$$
\begin{aligned}
& \int_{0}^{\infty} \int_{-\infty}^{\infty}\left|I_{4}\right|^{2} d \beta d x \\
& \leq O(1) \int_{0}^{\infty} \int_{-\infty}^{\infty}|\xi|^{-2} \mid\left(e^{\mu_{-}(\epsilon \xi) \frac{x}{\epsilon}}-e^{\frac{\lambda x}{a \epsilon}}\right) \\
& \times\left.\left(\tilde{n}_{1}(0, \xi)+\int_{0}^{\infty} e^{\xi \frac{y}{\lambda}} \partial_{y} \tilde{n}_{1}(y, \xi) d y\right)\right|^{2} d \beta d x \\
& \leq O(1) \int_{0}^{\infty} e^{2 \frac{\lambda x}{a \epsilon}} x^{2} d x \int_{-\infty}^{\infty} \mid \tilde{n}_{1}(0, \xi) \\
&+\left.\int_{0}^{\infty} e^{\xi \frac{y}{\lambda}} \partial_{y} \tilde{n}_{1}(y, \xi) d y\right|^{2} d \beta \\
& \leq O(1) \epsilon^{2}\|N\|_{1}^{2} .
\end{aligned}
$$

Combining all the estimates for $I_{k}, k=1,2,3$, 4 , we get

$$
\int_{0}^{\infty} \int_{0}^{\infty} e^{-2 \alpha t}\left|w^{\epsilon}-w-w^{B L}\right|^{2}(x, t) d x d t \leq O(1) \epsilon^{2}\|N\|_{1}^{2}
$$

The same analysis can be carried out as above on $z^{\epsilon}$, and we obtain

$$
\int_{0}^{\infty} \int_{0}^{\infty} e^{-2 \alpha t}\left|z^{\epsilon}-z\right|^{2}(x, t) d x d t \leq O(1) \epsilon^{2}\|N\|_{1}^{2}
$$

4.2. The case of uniform characteristic boundary. In the case of uniform characteristic boundary, namely $\lambda=0$, the asymptotics is a little different from the non-characteristic boundary case since the width of the boundary layer is of $O(1) \sqrt{\epsilon}$ order. The asymptotics (46) cannot catch the boundary layer in this case. Alternatively, we propose the following expansion:

$$
\left\{\begin{aligned}
w^{\epsilon}(x, t) & =w(x, t)+w^{B L}(y, t)+\sqrt{\epsilon} w_{1}^{B L}(y, t)+O(1) \epsilon \\
z^{\epsilon}(x, t) & =z(x, t)+z^{B L}(y, t)+\sqrt{\epsilon} z_{1}^{B L}(y, t)+O(1) \epsilon
\end{aligned}\right.
$$

where $w^{B L}(y, t), z^{B L}(y, t), w_{1}^{B L}(y, t)$, and $z_{1}^{B L}(y, t)$ are the boundary layers decaying exponentially fast in $y=x / \sqrt{\epsilon}$ when $y$ goes to infinity.

Plugging (46) into (25) and matching the expansion, we have the boundary layer problem

$$
\left\{\begin{aligned}
\partial_{t} w^{B L} & =a \partial_{y}^{2} z^{B L} \\
\partial_{y} z^{B L} & =0 \\
w^{B L}(y, 0) & =0 \\
z^{B L}(y, 0) & =0 \\
B_{u} w^{B L}(0, t) & =-B_{u} w(0, t) .
\end{aligned}\right.
$$


Therefore, the boundary layers are

$$
\begin{aligned}
\tilde{w}^{B L} & =-\frac{1}{\xi} e^{-\sqrt{\xi /(a \epsilon)} x} \tilde{n}_{1}(0, \xi) \\
\tilde{z}^{B L} & =0 .
\end{aligned}
$$

In this case, the boundary layer is different from that in the case of noncharacteristic boundary. We can found its effect both in $W_{I}^{\epsilon}+W_{I I}^{\epsilon}$ and $W_{\text {III }}^{\epsilon}$. First we consider $W_{I}^{\epsilon}+W_{I I}^{\epsilon}$. By integration by parts, we have

$$
\tilde{W}_{I}^{\epsilon}+\tilde{W}_{I I}^{\epsilon}-\tilde{W}-\frac{1}{2} \tilde{W}^{B L}=\left(I_{1}-\frac{1}{2} \tilde{W}^{B L}\right)+I_{2}+I_{3}+I_{4}
$$

where $I_{k}(k=1,2,3,4)$ denote the same items as in (45). According to the results of the asymptotic convergence in Section 3.2, we have

$$
\int_{-\infty}^{\infty} \int_{0}^{\infty}\left|I_{2}+I_{3}+I_{4}\right|^{2} d x d \beta \leq O(1) \epsilon\|N\|_{1}^{2}
$$

By using the estimate

$$
\left|I_{1}-\frac{1}{2} \tilde{W}^{B L}\right| \leq O(1)(1+x) \epsilon^{\frac{1}{2}} e^{R e \mu_{-}(\epsilon \xi) \frac{x}{\epsilon}}|\tilde{N}(0, \xi)|,
$$

we get

$$
\int_{-\infty}^{\infty} \int_{0}^{\infty}\left|I_{1}-\frac{1}{2} \tilde{W}^{B L}\right|^{2} d x d \beta \leq O(1) \epsilon^{\frac{3}{2}}\|N\|_{1}^{2}
$$

So far, we have established the result

$$
\int_{-\infty}^{\infty} \int_{0}^{\infty}\left|\tilde{W}_{I}^{\epsilon}+W_{I I}^{\epsilon}-\tilde{W}-\frac{1}{2} \tilde{W}^{B L}\right|^{2} d x d \beta \leq O(1) \epsilon\|N\|_{1}^{2}
$$

Next we consider $W_{\text {III }}^{\epsilon}$. By integration by parts, we write

$$
\begin{aligned}
\tilde{W}_{\text {III }}^{\epsilon}-\frac{1}{2} \tilde{W}^{B L}= & -\left(\begin{array}{c}
1 \\
g(\epsilon \xi)
\end{array}\right) \frac{e^{\mu_{-}(\epsilon \xi) \frac{x}{\epsilon}}\left(B_{u}+B_{v} k(\epsilon \xi)\right)}{2 \xi\left(B_{u}+B_{v} g(\epsilon \xi)\right)} \tilde{n}_{1}(0, \xi) \\
& +\left(\begin{array}{c}
1 \\
0
\end{array}\right) \frac{1}{2 \xi} e^{-\sqrt{\xi /(\epsilon \epsilon)} x} \tilde{n}_{1}(0, \xi) \\
& +\left(\begin{array}{c}
1 \\
g(\epsilon \xi)
\end{array}\right) \frac{e^{\mu_{-}(\epsilon \xi) \frac{x}{\epsilon}}\left(B_{u}+B_{v} k(\epsilon \xi)\right)}{2 a \xi\left(B_{u}+B_{v} g(\epsilon \xi)\right)} g(\epsilon \xi) \tilde{n}_{2}(0, \xi) \\
& -\left(\begin{array}{c}
1 \\
g(\epsilon \xi)
\end{array}\right) \frac{e^{\mu_{-}(\epsilon \xi) \frac{x}{\epsilon}}\left(B_{u}+B_{v} k(\epsilon \xi)\right)}{2 \xi\left(B_{u}+B_{v} g(\epsilon \xi)\right)} \tilde{s}^{\epsilon}(\xi) .
\end{aligned}
$$


We denote the two items on the right hand side of the above equality by $I_{1}, I_{2}$ and $I_{3}$, respectively. Noticing

$$
\left|I_{1}+I_{2}\right| \leq O(1) \epsilon^{\frac{1}{2}}(1+x) e^{R e \mu_{-}(\epsilon \xi) \frac{x}{\epsilon}}|\tilde{N}(0, \xi)|,
$$

we get

$$
\int_{-\infty}^{\infty} \int_{0}^{\infty}\left|I_{1}+I_{2}\right|^{2} d x d \beta \leq O(1) \epsilon^{\frac{3}{2}}\|N\|_{1}^{2}
$$

Similar to (37), we can prove that the following inequality holds for $I_{3}$ :

$$
\int_{-\infty}^{\infty} \int_{0}^{\infty}\left|I_{3}\right|^{2} d x d \beta \leq O(1) \epsilon\|N\|_{1}^{2}
$$

Therefore, we have

$$
\int_{0}^{\infty} \int_{0}^{\infty} e^{-2 \alpha t}\left|W_{\text {III }}^{\epsilon}-\frac{1}{2} W^{B L}\right|^{2}(x, t) d x d t \leq O(1) \epsilon\|N\|_{1}^{2} .
$$

Combining (51) and (52), we can establish the result

$$
\int_{0}^{\infty} \int_{0}^{\infty} e^{-2 \alpha t}\left|W^{\epsilon}-W-W^{B L}\right|^{2}(x, t) d x d t \leq O(1) \epsilon\|N\|_{1}^{2}
$$

Remark. If $N(x, t) \in H^{2}\left(\mathbb{R}^{+} \times \mathbb{R}^{+}\right)$, we can achieve a higher convergence rate by two times of integration by parts as follows.

$$
\int_{0}^{\infty} \int_{0}^{\infty} e^{-2 \alpha t}\left|W^{\epsilon}-W-W^{B L}\right|^{2}(x, t) d x d t \leq O(1) \epsilon^{\frac{3}{2}}\|N\|_{2}^{2}
$$

It is easy to verify the following relation between $U^{\epsilon}$ of the solution of (15) and $W^{\epsilon}$ of the solution of (21):

$$
U^{\epsilon}-U=W^{\epsilon}-W, \quad U^{I L}=W^{I L}, \quad U^{B L}=W^{B L} .
$$

Therefore, combining the results in [7] and the above arguments, we have the estimate

$$
\begin{aligned}
& \int_{0}^{\infty} \int_{0}^{\infty}\left|U^{\epsilon}-U-U^{I L}-U^{B L}\right|^{2}(x, t) e^{-2 \alpha t} d x d t \\
& \leq O(1) \epsilon^{2}\left\|W_{0}\right\|_{2}^{2}+ \begin{cases}O(1) \epsilon^{2}\left(\|c\|_{2}^{2}+\|N\|_{1}^{2}\right) & \text { if } \lambda \neq 0 \\
O(1) \epsilon^{3 / 2}\|c\|_{2}^{2}+\epsilon\|N\|_{1}^{2} & \text { if } \lambda=0\end{cases}
\end{aligned}
$$

Together with (26), we have proved that (10) is true. 


\section{Initial Boundary value problem: $n>1$}

In this section we briefly give the idea of the proof of Theorem 1.2. For the case $n>1$, first we take the change of variables according to (16) and (17) - (20). Thanks to [7], we only have to deal with the following system:

$$
\left\{\begin{aligned}
\partial_{t} W^{\epsilon}+A \partial_{x} W^{\epsilon} & =\frac{1}{\epsilon} S W^{\epsilon}+N \\
W(x, 0) & =0 \\
B W(0, t) & =0
\end{aligned}\right.
$$

By using the Laplace transform in (56), we get

$$
\begin{aligned}
\tilde{W}^{\epsilon}= & \left(\begin{array}{c}
I \\
K(\epsilon \xi)
\end{array}\right)(G(\epsilon \xi)-K(\epsilon \xi))^{-1} \\
& \times \int_{x}^{\infty} e^{\mu_{+}(\epsilon \xi) \frac{(x-y)}{\epsilon}}\left(L N_{1}(y, \xi)-\frac{1}{a} G(\epsilon \xi) L N_{2}(y, \xi)\right) d y \\
& +\left(\begin{array}{c}
I \\
G(\epsilon \xi)
\end{array}\right)(G(\epsilon \xi)-K(\epsilon \xi))^{-1} \\
& \times \int_{0}^{x} e^{\mu_{-}(\epsilon \xi) \frac{(x-y)}{\epsilon}}\left(L N_{1}(y, \xi)-\frac{1}{a} K(\epsilon \xi) L N_{2}(y, \xi)\right) d y \\
& -\left(\begin{array}{c}
I_{n} \\
G(\epsilon \xi)
\end{array}\right) e^{\mu_{-}(\epsilon \xi) \frac{x}{\epsilon}}\left(B_{u}+B_{v} G(\epsilon \xi)\right)^{-1}\left(B_{u}+B_{v} K(\epsilon \xi)\right) \\
& \times(G(\epsilon \xi)-K(\epsilon \xi))^{-1} \tilde{W}^{\epsilon}(\xi)
\end{aligned}
$$

where

$$
\begin{aligned}
\mu_{ \pm}(\xi) & =\operatorname{diag}\left\{\mu_{1}^{ \pm}(\xi), \mu_{2}^{ \pm}(\xi), \cdots, \mu_{n}^{ \pm}(\xi)\right\} \\
G(\xi) & =\operatorname{diag}\left\{g_{1}(\xi), g_{2}(\xi), \cdots, g_{n}(\xi)\right\} \\
K(\xi) & =\operatorname{diag}\left\{k_{1}(\xi), k_{2}(\xi), \cdots, k_{n}(\xi)\right\} \\
\tilde{R}^{\epsilon}(\xi) & =\int_{0}^{\infty} e^{-\mu_{+}(\epsilon \xi) \frac{y}{\epsilon}}\left(L N_{1}(y, \xi)-\frac{1}{a} G(\epsilon \xi) L N_{1}(y, \xi)\right) d y
\end{aligned}
$$

with

$$
\mu_{j}^{ \pm}(\xi)=\frac{1}{2 a}\left(\lambda_{j} \pm \sqrt{\lambda_{j}^{2}+4 a \xi(1+\xi)}\right), g_{j}(\xi)=\frac{\mu_{j}^{+}(\xi)}{1+\xi}, k_{j}(\xi)=\frac{\mu_{j}^{-}(\xi)}{1+\xi} .
$$

Since all the components of $\tilde{W}^{\epsilon}$ can be treated seperately, similar to the scalar case, we can prove that (13) and (14) are true. Here we omit the details.

Remark. As we can see that the convergence rate in this case is lower than that in the scalar case. It is because that the non-characteristic boundary layers and the uniform characteristic boundary layers are mixed up, therefore the best convergence rate we can achieve is the rate of the uniform characteristic boundary case. 
Acknowledgement. The substantial influence of Xin and $\mathrm{Xu}$ [7] on this paper is clear. This work was partially supported by the NSFC and the Educational Ministry of China.

\section{References}

[1] Chen, G.-Q., Levermore, C.D. and T.-P. Liu: Hyperbolic conservation laws with stiff relaxation terms and entropy. Comm. Pure Appl. Math. 47 (1994), $787-830$.

[2] Higdon, R.L.: Initial boundary value problems for linear hyperbolic systems. SIAM Rev. 28 (1986), 177 - 217.

[3] Jin, S. and Z. Xin: The relaxation schemes for systems of conservation laws in arbitrary space dimensions. Comm. Pure Appl. Math. 48 (1995), 235 - 277.

[4] Natalini, R.: Recent results on hyperbolic relaxation problems. In: Analysis of Systems of Conservation Laws (ed.: H.Freistühler). Monogr. Surv. Pure and Appl. Math., Vol. 99. Boca Raton: Chapman \& Hall/CRC 1998, pp. 128-198.

[5] Natalini, R.: Convergence to equilibrium for the relaxation approximations of conservation laws. Comm. Pure Appl. Math. 49 (1996), 795 - 822.

[6] Wang, W.-C. and Z. Xin: Asymptotic limit of initial boundary value problems for conservation laws with relaxational extensions. Comm. Pure Appl. Math. 51 (1998), $505-535$.

[7] Xin, Z. and W.-Q. Xu: Stiff well-posedness and asymptotic convergence for a class of linear relaxation systems in a quarter plane. J. Diff. Eqs. 167 (2000), $388-437$.

[8] Xu, W.-Q.: Relaxation limit for piecewise smooth solution to system of conservation laws. J. Diff. Eqs. 162 (2000), 140 - 172.

[9] Yong, W.-A.: Boundary conditions for hyperbolic systems with stiff source terms. Indiana Univ. Math. J. 48 (1999), 115 - 137.

Received 05.05.2003; in revised form 02.03.2004 